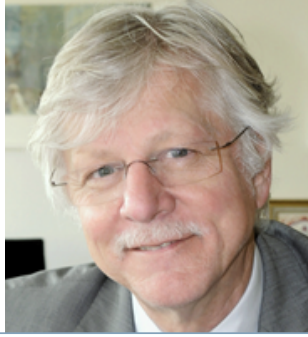

Wilfried Müller

\title{
Leitziel „Eigenständiges Lernen“: perspektivenreich und realistisch
}

In den letzten Jahren hat es an den Hochschulen, in den Medien und in der Politik eine kontroverse Debatte über die „Qualität der Lehre" gegeben, deren Intensität durch die studentischen Proteste des letzten Winters verstärkt wurde. Können wir heute sagen, was „gute Lehre“ über die Fachgrenzen hinweg bedeutet? Die Antwort lautet „ja“- sofern man sich auf den aktuellen Stand der empirischen Lehr- und Lernforschung einlässt. Und diese besagt, dass das Lernergebnis im Wesentlichen von den Lehrstrategien der Lehrenden abhängt. Nicht die verwendeten Medien beeinflussen die Lernleistungen, sondern die didaktische Gestaltung der Lernumgebungen. Vor diesem Hintergrund hat die Hochschulrektorenkonferenz im April 2008 in ihrer Empfehlung „Für eine Reform der Lehre in den Hochschulen“ mit Unterstützung deutscher Lehr- und Lernforscher formuliert: „Qualität der Lehre bemisst sich daran, wieweit es den Lehrenden gelingt, ihre Studierenden zu ermutigen und zu befähigen, eigenständig zu lernen“. Diese Definition greift insofern den im Bologna-Prozess postulierten Shift from Teaching to Learning auf, als es den Erfolg der Lehre daran bemisst, was die Studierenden gelernt haben.

Die Praktiker des Hochschulunterrichts werden sich fragen, ob dieser Anspruch unter den Alltagsbedingungen deutscher Hochschulen umgesetzt werden kann. Unberechtigt ist diese Skepsis angesichts der relativ schlechten personellen Ausstattung nicht. Aber: Man kann mit dieser Definition auch unter erschwerten Bedingungen etwas anfangen, wenn man dieses Plädoyer für das eigenständige Lernen als Leitziel versteht, das uns sagt, in welche Richtung wir schauen und vor allem gehen sollen.

Im Folgenden einige Anregungen, was Ermutigung und Befähigung zum eigenständigen Lernen unter den heutigen Hochschulverhältnissen konkret bedeuten kann. Dabei liegt der Schwerpunkt auf dem Hochschulunterricht in der Bachelorphase:

Erstens: Eine wesentliche Voraussetzung für die Umsetzung dieses Leitziels ist eine gute Betreuungsrelation zwischen Studierenden und Lehrenden. Hierzu sind mehr Stellen für den wissenschaftlichen Mittelbau erforderlich. In großen Veranstaltungen ist der Dialog mit den Studierenden schwieriger als in kleinen Seminaren. Aber gute Betreuungsrelationen sind keine Garantie für „gute Lehre“: Es kommt schon auf die Strategie und den Stil der Lehrenden an.

Zweitens: Es sollte den Studierenden die Chance zur Entwicklung einer eigenen Lernstrategie gegeben werden. Hierzu muss man sie beraten, aber ihren Lernweg müssen sie letztlich selbst finden. Im Wahlpflichtbereich sollten sie die Chance haben, individuelle Profile zu bilden, etwa sich zwischen Meeresbiologie, Neurobiologie oder Ökologie zu entscheiden. $\mathrm{Zu}$ geringe Wahlfreiheiten sind nicht förderlich für ein eigenständiges Lernverhalten, zu große Wahlfreiheiten können Studierende überfordern. Es kommt auf das richtige Maß an.

Drittens: Vermutlich besteht der entscheidende Anstoß zum eigenständigen Lernen darin, den Studierenden trotz aller personellen Engpässe ein- oder zweimal im BachelorStudium die Chance zum forschenden Lernen zu geben, beispielsweise in einem Projekt über Umweltwirkungen von Nanomaterialien. Entscheidend daran ist die eigenständige studentische Reflexion über Ziele, Methoden, Ergebnisse des Projekts etc.

Viertens: Das regelmäßige Feedback über den Stand der Lernbemühungen - auch jenseits der „offiziellen Prüfungen“ - kann als der Schlüssel zur Unterstützung eines aktiven selbstständigen Lernens betrachtet werden - eine Rückmeldung also darüber, was die Studierenden gut gemacht haben und was sie noch tun müssen, um sich zu verbessern.
Vor diesem Hintergrund ist es sehr wichtig, den Studierenden Prüfungsformen anzubieten, in denen sie nicht nur Wissen reproduzieren, sondern ihre Kompetenzen im Umgang mit dem erworbenen Wissen dokumentieren müssen, etwa Vorträge, Hausarbeiten und Projektberichte.

Fünftens: Auch von den Studierenden wird verlangt, dass sie eine Mit-Verantwortung für ihren Lernerfolg übernehmen. Hierzu gehören kontinuierliche Mitarbeit, Engagement, Interesse und Neugier im und für das Studium.

Last but not least: Es muss viel mehr als bisher für die hochschuldidaktische Weiterbildung der Lehrenden getan werden, um den Shift from Teaching to Learning im Alltag vollziehen zu können. Viele Fachbereiche und Fakultäten haben sich auf den Weg in diese Richtung gemacht. Noch aber fehlt der große Durchbruch. Der von Bund und Ländern ausgeschriebene Qualitätspakt mit einer jährlichen Förderung von 200 Millionen Euro könnte das bisher fehlende Momentum erzeugen.

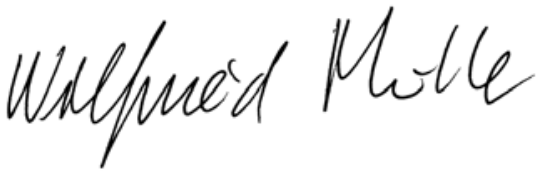

Wilfried Müller,

Rektor der Universität Bremen,

Vizepräsident der

Hochschulrektorenkonferenz

Korrespondenzadresse: Prof. Dr. Wilfried Müller Universität Bremen Bibliothekstraße 1 D-28359 Bremen Tel.: 0421-218-60011 Fax: 0421-218-42 59 rektor@uni-bremen.de 